\title{
Revista de Enfermagem
}

\section{SAÚDE DO HOMEM E DETERMINANTES SOCIAIS NA SAÚDE COLETIVA \\ MEN'S HEALTH AND SOCIAL DETERMINANTS IN COLLECTIVE HEALTH \\ DETERMINANTES SOCIALES Y DE LA SALUD DEL HOMBRE EN LA SALUD COLECTIVA}

Jessica Lorena Palmeira de Morais ${ }^{1}$, Karelline Izaltemberg Vasconcelos Rosenstock ${ }^{2} \oplus$, Catarina Maria Andrade Figueiredo Guimarães Maia ${ }^{3}{ }^{\circledR}$, Jancelice dos Santos Santana ${ }^{4}$ (i)

\section{RESUMO}

Objetivo: verificar a produção científica online acerca da atenção à saúde do homem sob a luz da reflexão sobre seus determinantes sociais no âmbito da saúde coletiva. Método: trata-se de um estudo bibliográfico, tipo revisão integrativa de abordagem qualitativa, descritiva e exploratória, realizado na Biblioteca Virtual em Saúde (BVS) e no Google Acadêmico, com recorte temporal de 2005 a 2019, desenvolvido entre janeiro e maio de 2019. Analisaram-se os estudos em seis fases, tendo sido apresentados em forma de figuras e sínteses. Resultados: constatou-se que os determinantes sociais são, em grande parte, responsáveis pela iniquidade no acesso aos serviços de saúde. $\mathrm{O}$ homem tem dificuldade em reconhecer suas necessidades voltadas à saúde, mascarando suas fragilidades e não considerando a prevenção da saúde um critério predominante em suas vidas por não perceberem o cuidado como uma prática masculina. Considerações finais: observou-se a necessidade de que os homens reflitam sobre a masculinidade para a compreensão dos comprometimentos de sua saúde.

Descritores: Determinantes Sociais de Saúde; Saúde do Homem; Saúde Coletiva; Atenção à Saúde; Prevenção de Doenças; Centros de Saúde.

\section{ABSTRACT}

Objective: to verify the online scientific production on men's health care under the light of the reflection on its social determinants in the scope of collective health. Method: this is a bibliographical, integrative review type study of qualitative, descriptive and exploratory approach, carried out in the Virtual Health Library (VHL) and on Google Scholar, within the time frame from 2005 to 2019, developed between January and May 2019. The studies were analyzed in six phases, being presented in the form of figures and summaries. Results: social determinants were found to be 
largely responsible for the inequity in the access to health services. Men have difficulty in recognizing their health needs, masking their weaknesses and not considering health prevention as a predominant criterion in their lives because they do not perceive health care as a male practice. Final considerations: the need for men to reflect on masculinity in order to understand the compromises to their health was observed.

Descriptors: Social Determinants of Health; Men`s Care; Public Health; Delivery of Health Care; Disease Prevention; Health Centers.

\section{RESUMEN}

Objetivo: verificar la producción científica online sobre la atención a la salud del hombre a la luz de la reflexión de sus determinantes sociales en el ámbito de la salud pública. Método: se trata de un estudio bibliográfico, tipo revisión integradora con abordaje cualitativo, descriptivo y exploratorio realizado en la Biblioteca Virtual en Salud (BVS) y Google Académico, con un marco temporal de 2005 a 2019, desarrollado entre enero y mayo de 2019. Los estudios se analizaron en seis fases que se presentaron en forma de figuras y resúmenes. Resultados: se encontró que los determinantes sociales son en gran parte responsables del acceso inequitativo a los servicios de salud. A los hombres les cuesta reconocer sus necesidades relacionadas con la salud, pero enfrentan sus debilidades y porque consideran que el cuidado no es una práctica masculina, no considerando la prevención de la salud como un criterio predominante en sus vidas. Consideraciones finales: era necesario que los hombres reflexionaran sobre la masculinidad para comprender los deterioros de su salud.

Descriptores: Determinantes Sociales de la Salud; Salud del Hombre; Salud Pública; Atención a la Salud; Prevención de Enfermedades; Centros de Salud.

\footnotetext{
1,2,3,4 Centro Universitário UNIESP. Cabedelo (PB), Brasil.

1 (i) https: / / orcid.org/0000-0002-2114-2054

2 ii https: / / orcid.org/0000-0002-9305-1362

3 ii https: / / orcid.org/0000-0003-4314-0911

4 (i) https://orcid.org/0000-0002-1062-8518
}

\section{Como citar este artigo}

Morais JLP, Rosenstock KIV, Maia CMAFG, Santana JS. Saúde do homem e determinantes sociais na saúde coletiva. Rev enferm UFPE on line. 2021;15(2):e247844 DOI: https://doi.org/10.5205/1981-8963.2021.247844 


\section{INTRODUÇÃO}

Devido ao contexto cultural perpassado desde a antiguidade, os homens foram ensinados a acreditar que são seres mais fortes do que as mulheres, seja nos aspectos físico, emocional, psicológico, bem como no quesito saúde. ${ }^{1}$ Dessa forma, acreditando serem mais sadios e resistentes, é natural que a busca pela medicina preventiva nos cuidados à saúde do homem seja defasada e menor em comparação aos cuidados realizados para as mulheres. Por conseguinte, os homens são mais vulneráveis a contrair doenças do que as mulheres. ${ }^{2}$

Assim, instituída pela Portaria GM/MS $\mathrm{n}^{\circ} 1.944$ de 27 de agosto de 2009, surge a Política Nacional de Atenção Integral à Saúde do Homem (PNAISH), que tem como objetivo facilitar e ampliar o acesso com qualidade da população masculina na faixa etária de 20 a 59 anos às ações e aos serviços de assistência integral à saúde da Rede Sistema Único de Saúde, mediante atuação nos aspectos socioculturais, sob a perspectiva relacional de gênero e dentro da lógica da concepção de linhas de cuidado que respeitem a integralidade da atenção, contribuindo de modo efetivo para a redução da morbimortalidade e para melhores condições de saúde desta população. ${ }^{3}$

As diferenças de morbimortalidade entre homens e mulheres são amplamente conhecidas: os homens morrem mais cedo, principalmente por causas externas, acidentes e violência; são mais suscetíveis às doenças cardiovasculares, possivelmente por comportamentos de risco mais frequentes, bem como a ausência de cuidados preventivos com a saúde. Cabe ressaltar que a utilização de serviços de saúde pela população é influenciada por diferentes fatores, como a necessidade e disponibilidade de serviços de saúde, a propensão da população em utilizá-los, e a facilidade de acesso. ${ }^{4}$

Nesse aspecto, um importante fator a ser considerado são os Determinantes Sociais da Saúde (DSS) que, de acordo com a Organização Mundial de Saúde,$^{5}$ estão relacionados às condições em que uma pessoa vive e trabalha, incluindo os fatores sociais, econômicos, culturais, étnicos/raciais, psicológicos e comportamentais que influenciam a ocorrência de problemas de saúde, além de fatores de risco à população, tais como moradia, alimentação, escolaridade, renda e emprego.

Portanto, os determinantes sociais na saúde do homem não podem ser avaliados somente pelas doenças que acometem essa parcela da população, pois perpassam várias dimensões do processo de saúde tanto do ponto de vista do indivíduo quanto da coletividade na qual ele se insere. Entre os desafios para entender a relação entre determinantes sociais e a saúde do homem está o estabelecimento de uma hierarquia de determinações entre os fatores mais gerais de 
natureza social, econômica e política, e as mediações através das quais esses fatores incidem sobre a situação de saúde desse grupo, não havendo uma simples relação direta de causa-efeito. ${ }^{6}$

Alguns estudos relatam a invisibilidade dos homens na atenção primária à saúde, uma vez que, historicamente, esses serviços têm desenvolvido mais ações destinadas à saúde de mulheres, crianças e idosos. A ausência dos homens nas UBS pode ser explicada por elas não disponibilizarem atividades ou programas direcionados especificamente para esse público, além do fato de que os homens preferem utilizar serviços que respondem mais rápida e objetivamente às suas demandas, como farmácia e pronto socorro..$^{8-9-10,7}$

Com o intuito de subsidiar a reflexão acerca da saúde do homem e a correlação dos determinantes sociais diante do absentismo desse grupo na Atenção Primária, o presente estudo apontará os motivos que acarretam essa ausência por parte dos usuários do sexo masculino e as dificuldades apresentadas pela equipe de saúde na prestação dos cuidados ao homem que dificilmente comparece à Unidade Básica de Saúde (UBS) para prevenção de doenças e agravos.

Diante de tais constatações, surgiu o seguinte questionamento: Como se configura a produção científica online acerca da atenção à saúde do homem sob a luz da reflexão sobre seus determinantes sociais no âmbito da saúde coletiva?

\section{OBJETIVO}

Verificar a produção científica online acerca da atenção à saúde do homem sob a luz da reflexão sobre seus determinantes sociais no âmbito da saúde coletiva.

\section{MÉTODO}

A fim de alcançar o objetivo proposto, fez-se um estudo bibliográfico, tipo revisão integrativa da literatura, de abordagem qualitativa, descritiva e exploratória. A pesquisa de abordagem qualitativa está fundamentada no conhecimento sobre as pessoas a partir da descrição da experiência humana, propiciando um campo livre ao rico potencial das percepções e subjetividades dos seres humanos. ${ }^{11-12}$ A pesquisa descritiva observa, registra, analisa e correlaciona fenômenos (ou fatos) do mundo físico e especialmente do mundo humano que ocorrem na vida social, política e econômica do indivíduo tomado isoladamente, assim como de grupos e comunidades mais complexas. A pesquisa exploratória tem por objetivo conhecer a variável do estudo tal como se apresenta, seu significado e o contexto no qual ela se insere, pressupondo-se que o comportamento humano é melhor compreendido no contexto social em que ocorre. ${ }^{13}$ 
A revisão integrativa da literatura é um método específico que resume o passado da literatura empírica ou teórica para fornecer uma compreensão mais abrangente de um fenômeno particular. Esse método de pesquisa objetiva traçar uma análise sobre o conhecimento já construído em pesquisas anteriores sobre um determinado tema. Ela possibilita a síntese de vários estudos já publicados, permitindo a geração de novos conhecimentos pautados em resultados apresentados por pesquisas anteriores. ${ }^{11}$

Assim, neste estudo, foram adotadas as seguintes etapas: ${ }^{11}$ identificação do tema e seleção da questão de pesquisa; estabelecimento de critérios de inclusão e exclusão; identificação dos estudos pré-selecionados e selecionados, bem como a categorização dos estudos selecionados; análise e interpretação dos resultados; e apresentação da revisão/síntese do conhecimento.

As sínteses qualitativas têm início com uma boa pergunta de pesquisa analítica que deve investigar a relação entre dois eventos, sendo formada por diversos componentes relacionados no anagrama PICo: Participantes; fenômeno de Interesse (ou exposição); e Contexto do estudo. Assim, os Participantes correspondem ao grupo a ser observado no estudo; o fenômeno de Interesse indica o que deve ser observado na pesquisa em determinados participantes, e o Contexto do estudo representa o que se espera ao final da revisão integrativa, indicando as áreas a serem selecionadas como estratégia de busca de possíveis estudos que se adequem aos objetivos da pesquisa. Para este estudo, foi formulada a seguinte questão de pesquisa (QP): como se configura a produção científica online acerca da atenção à saúde do homem sob a luz da reflexão sobre seus determinantes sociais no âmbito da saúde coletiva?

A QP foi estruturada da seguinte maneira:

- Participantes: pesquisas disponíveis online acerca da atenção à saúde do homem e seus determinantes sociais;

- Fenômeno de Interesse: atenção à saúde do homem e seus determinantes sociais;

- Contexto do estudo: estratégias e abordagens da atenção à saúde do homem no âmbito da saúde coletiva.

Para a seleção do material de estudo, as principais fontes de busca e pesquisa consultadas foram a Biblioteca Virtual em Saúde (BVS), selecionando as bases de dados LILACS (Literatura Latino-Americana e do Caribe em Ciências da Saúde), BDENF (Base de Dados de Enfermagem), OPAS (Acervo da Biblioteca da Organização Pan-Americana de Saúde) e WHOLIS (Sistema de Informação da Biblioteca da OMS), além do Google Acadêmico, pois possuem um número significativo de publicações latino-americanas na área do tema desta pesquisa. Para a busca automática, foram empregados os seguintes descritores: determinantes sociais, saúde do homem, saúde coletiva. 
Critérios de inclusão $(\mathrm{Cl})$ : qualquer artigo científico que aborde ou discuta (direta ou indiretamente) os determinantes sociais e a atenção à saúde do homem no âmbito da saúde coletiva; manuscrito que esteja completamente disponível na Web e publicado no idioma português no período compreendido entre 2005 e 2019.

Critérios de exclusão (CE): estudos que lidem com ou mencionem a saúde do homem sem abordar os determinantes sociais ou a saúde coletiva; estudos escritos em idioma diferente do português; versão completa do manuscrito que não esteja disponível na Web; ou artigos publicados fora do período compreendido entre 2005 e 2019.

As buscas nas bibliotecas de pesquisa selecionadas foram realizadas entre janeiro e maio de 2019. Na busca automática, após obter o resultado da pesquisa, os pesquisadores realizaram a leitura dos títulos e abstracts dos manuscritos encontrados a fim de verificar se os participantes e o fenômeno de interesse atendiam à questão norteadora. Desta forma, 42 estudos atendiam aos $\mathrm{Cl}$ e CE deste estudo, sendo realizado o download do arquivo do tipo bibtex contendo o título e o abstract de cada publicação por meio da funcionalidade de exportação disponível nessas bibliotecas. Em seguida, as publicações selecionadas foram agrupadas, excluindo-se estudos duplicados de cada retorno.

Após a leitura, 25 estudos foram excluídos pelos seguintes motivos: 3 foram resultados de trabalho de conclusão de curso de graduação; 9 dissertações de mestrado na íntegra; e 13 abordavam apenas a saúde do homem, sem mencionar os determinantes sociais nem a saúde coletiva. Assim, as 17 publicações incluídas na etapa final foram lidas na íntegra para preenchimento do instrumento-roteiro, no intuito de garantir a elegibilidade dos estudos selecionados para compor a amostra final. 0 instrumento-roteiro foi composto pelos seguintes campos: título do artigo, autores, ano de publicação, idioma, base de dados, área do conhecimento de desenvolvimento do estudo, tipo de estudo, objetivo, características metodológicas, principais aspectos da análise de dados, resultados relevantes, e principais contribuições com o tema. 0 processo de seleção das publicações está apresentado na Figura 1. 

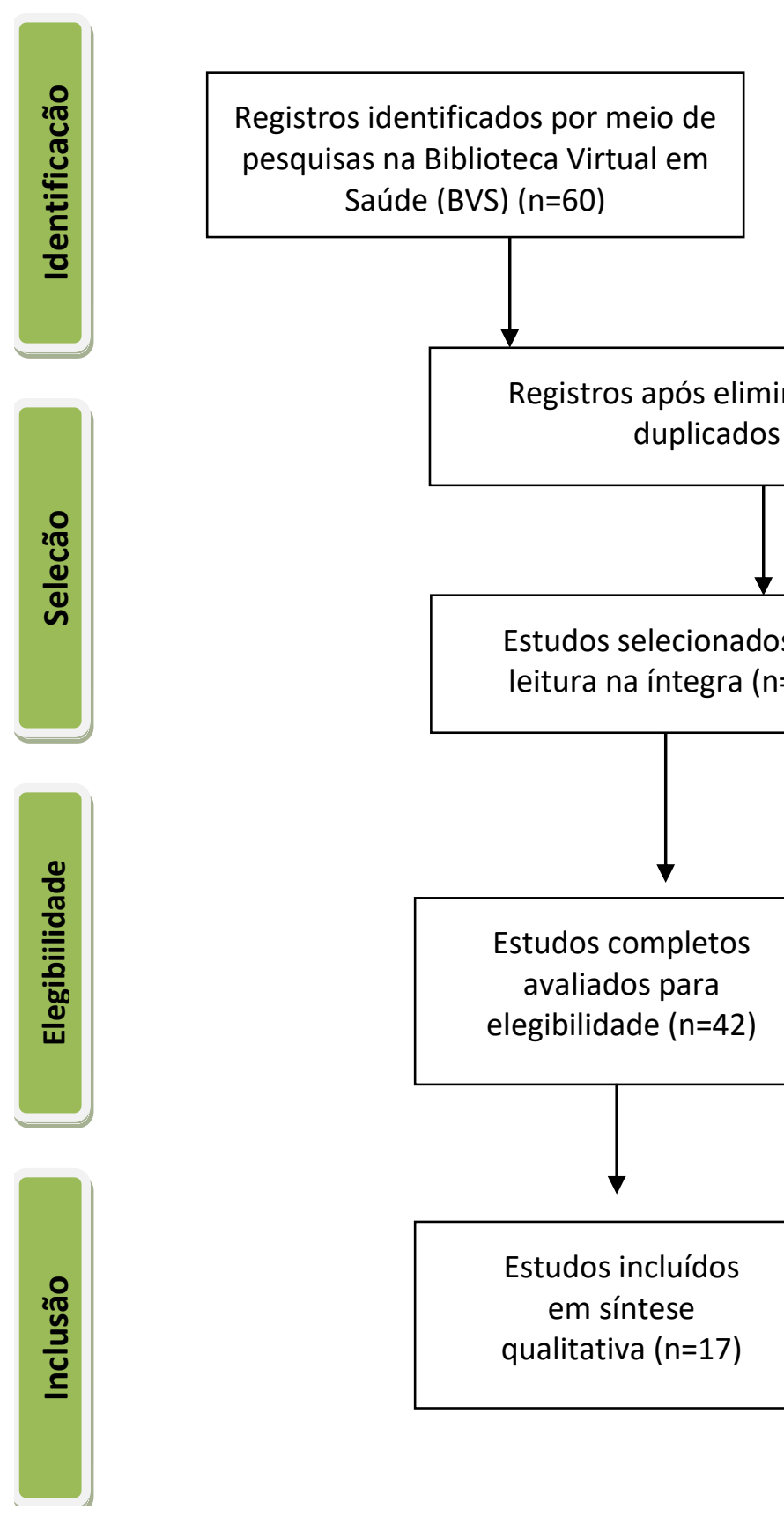

Figura 1. Fluxograma da seleção dos estudos, adaptado do Preferred Reporting Items for Systematic Reviews and MetaAnalyses (PRISMA, 2009). Cabedelo (PB), Brasil, 2020.

\section{RESULTADOS}

Após o processo e a busca na base de dados, selecionou-se o total de 17 publicações. Os achados foram organizados na forma de quadro para melhor identificação de cada publicação, selecionada com as seguintes informações: identificação alfanumérica de A1 a A17, incluindo nome do artigo, periódico, ano de publicação, local, objetivo, método, e resultados, conforme apresentado na Figura 2. ic pesquisas em outras fontes de dados (Google Acadêmico) ( $n=132$ ) 
A1 A visão dos profissionais Analisar as concepções que Ausência de ações direcionadas sobre a presença e as os profissionais da saúde para o público masculino da demandas dos homens possuem sobre as demandas faixa etária de 20 a 59 anos, nos serviços de saúde: e os comportamentos bem como a dificuldade em perspectivas para a específicos da população faltar ao trabalho para ir às análise da implantação masculina atendida nos consultas.

da Política Nacional de serviços de saúde.

Atenção Integral à Saúde do Homem. ${ }^{14}$

Pesquisa quanti-qualitativa, estudo de caso.

Revista Ciência \& Saúde

Coletiva 2012,

Brasília/DF, Brasil.

A2 A saúde do homem na Conhecer e analisar a visão Ausência de infraestrutura visão dos enfermeiros de dos enfermeiros em relação organizacional uma Unidade Básica de ao atendimento à saúde do sistematização dos serviços Saúde. ${ }^{15}$ homem. Pesquisa qualitativa para atender às necessidades

Escola Anna Nery, 2012, Rio de Janeiro/RJ, Brasil. que teve como campo um do gênero masculino.

Centro Municipal de Saúde.

Pesquisa qualitativa.

A3 A produção científica Analisar artigos de pesquisas Existência de importantes sobre acesso no âmbito existentes e relatos de lacunas e necessidades a serem do Sistema Único de experiência tematizando o atendidas na qualidade da Saúde do Brasil: avanços, acesso ao SUS. atenção e no acesso aos limites e desafios. ${ }^{16}$ serviços em nível de atenção

Saúde e Sociedade, 2014, Pesquisa qualitativa. básica.

Brasil.

Atenção à saúde do Descrever a vivência de Por um lado, déficit de homem: interlocução ações de educação em conhecimento por parte da entre ensino e serviço. ${ }^{17}$ saúde desenvolvidas pelo população masculina em Revista Acta Paulista de Grupo de Estudos e relação à promoção da saúde e Enfermagem, 2011, João Pessoa/PB, Brasil. Pesquisas sobre à prevenção de agravos; e, por Masculinidades e Saúde da outro lado, o grande desafio Universidade Federal da para implementar ações de Paraíba durante a primeira educação que visem a romper Semana Estadual de Atenção os déficits de autocuidado à Saúde do Homem. nesses indivíduos, bem como nos exercentes da área da descritivo, saúde. 
qualitativo, do tipo relato

de experiência.

A5 Assistência Integral à Conhecer as necessidades A criação da Política de Saúde do Homem: de saúde, identificar os Atenção Integral à Saúde do necessidades, obstáculos obstáculos que impedem o Homem não foi o suficiente e estratégias de atendimento das para inseri-lo no contexto de enfrentamento. ${ }^{18}$ necessidades de saúde do saúde.

Escola Anna Nery, 2014, homem e apresentar Cuité/PB, Brasil. estratégias de enfrentamento.

Pesquisa descritiva,
exploratória, com
abordagem qualitativa.

Determinante de saúde Identificar os determinantes Os determinantes sociais de no Brasil: a procura da de saúde de maior impacto saúde são em grande parte os equidade na saúde. ${ }^{19}$ na saúde da população. responsáveis pela iniquidade

Saúde e Sociedade, 2017, Brasil-BRA.

Pesquisa descritiva com saúde. no acesso aos cuidados de recurso de análise

bibliométrica.

A7 Atenção Integral à Saúde Compreender a percepção Déficit de conhecimento do do Homem: um desafio dos profissionais de saúde PNAISH por parte dos na atenção básica. ${ }^{20}$ sobre a Política Nacional de profissionais e baixa

Revista Brasileira em Promoção da Saúde, 2016, Quixadá/CE, Brasil.
Atenção Integral à Saúde do compreensão da sua Homem (PNAISH).

Pesquisa qualitativa e

descritiva.

A saúde e seus Analisar as relações entre Ressalta-se a importância da determinantes sociais. ${ }^{21}$ saúde e seus determinantes adoção de políticas públicas Revista Saúde Coletiva, sociais.

2007, Rio de Janeiro/RJ, Brasil. para a promoção da equidade em saúde.

Pesquisa qualitativa.

A9 Saúde do homem e Analisar a saúde do homem Prevalecem o estereótipo da masculinidades na e masculinidades na PNAISH, sexualidade masculina e o Política Nacional de à luz da reflexão que estes pensamento sob a ótica Atenção Integral à Saúde temas receberam nos masculina de que membros do do Homem: uma revisão últimos tempos na literatura sexo masculino não adoecem, bibliográfica. ${ }^{22}$ da Saúde Coletiva/Saúde não necessitando, assim, de 
Pública.

Saúde e Sociedade, 2013,

Brasil-BRA.

Análise crítica da literatura.

A10 Gênero e saúde: o cuidar Analisar a percepção dos As práticas preventivas não do homem em debate. ${ }^{23}$ homens sobre os cuidados fazem parte do cotidiano da Revista Psicologia - com a própria saúde. população masculina, Teoria e Prática, 2011, Campina Grande/PB, Brasil.

Estudo de caráter concepções machistas, que se transversal, descritivo e considera invulnerável no analítico, com abordagem quesito doenças. qualitativa.

A11 O homem na atenção Compreender a percepção Presença de fragilidades na básica: percepções de de enfermeiros sobre as atuação dos serviços de saúde enfermeiros sobre as implicações das questões de perante o público masculino. implicações do gênero na gênero na saúde do homem saúde. ${ }^{24}$ e na oferta de serviços a

Escola Anna Nery, 2014, esse público. Juazeiro do Norte/CE, Brasil.

Estudo descritivo, com abordagem qualitativa.

A12 A Política Nacional de Avaliar o que pensam os Constatou-se que o público Saúde do Homem: homens a respeito da masculino não percebe a necessidade ou ilusão? ${ }^{25}$ criação de uma Política implantação de uma política Revista Psicologia Nacional voltada para a de saúde específica para o 2010, saúde masculina.

Vitória/ES, Brasil. homem como um benefício para eles mesmos.

Pesquisa qualitativa, estudo de caso.

A13 Política de saúde do Discutir a articulação entre Observou-se o aumento da homem. ${ }^{26}$ sistemas de informação notoriedade da saúde da epidemiológica, produção população masculina nos

Revista Saúde Pública, 2012, São Paulo, Brasil. científica, e políticas das últimos anos por meio da ações voltadas para a divulgação e exploração dos assistência à saúde do dados dos sistemas de homem. informações epidemiológicas e da maior produção científica,

Levantamento contribuindo para a formulação epidemiológico bibliográfico. e de ações, estratégias e políticas governamentais. homem: ações e organização dos serviços saúde, em especial 
perspectivas

enfermeiros. ${ }^{27}$

Revista Mineira de

Enfermagem, Recife/PE, Brasil.

dos desenvolvidos no âmbito da enfermeiras, para lidar com a ESF e ao planejamento de saúde do homem, afirmando ações de promoção da saúde não estarem preparadas. que contemplem população masculina.
Pesquisa descritiva e masculinidade ao não

a Salienta-se a impaciência dos pacientes homens no quesito espera, bem como no fator da exploratória de abordagem acreditarem em saúde qualitativa.

preventiva.

A15 Saúde dos homens: Analisar como a temática Observou-se uma abordagem abordagem na formação relacionada à saúde dos generalizada em relação à de enfermeiros. ${ }^{28}$ homens é abordada nos saúde dos homens e à inserção currículos dos cursos de da Política Nacional de

Revista de Enfermagem UERJ, 2014, Rio Grande do Sul/RS, Brasil. graduação em Enfermagem Atenção Integral à Saúde do em um município do Rio Homem nas discussões Grande do Sul/RS, Brasil. acadêmicas durante a graduação em enfermagem.

Pesquisa documental de abordagem qualitativa.

Atenção à saúde do Analisar as ações de atenção Os enfermeiros consideram homem em Unidades de à saúde do homem importante a política de saúde Estratégia de Saúde da realizadas por enfermeiros do homem, porém ainda são Família. ${ }^{29}$ em Unidades de Estratégia frágeis as condições de Revista Enfermagem UFSM, 2011, Vale do Rio Pardo/RS, Brasil. de Saúde da Família e as implementação dessa política, condições de informação em especial de conhecimento, desses profissionais sobre a incentivo e planejamento de Política Integral de Saúde do ações específicas destinadas ao Homem em dois municípios homem.

em gestão plena do Vale do

Rio Pardo/RS.

Pesquisa qualitativa de caráter exploratóriodescritivo.

A17 Determinantes sociais da Destacar os reducionismos Nesse sentido, entendemos saúde: o "social" em cada vez mais presentes na configurar-se o campo dos questão. 30 abordagem do social no determinantes sociais da

Saúde e Sociedade, 2014, campo dos determinantes saúde, já que se encontra sociais da saúde.

Estudo exploratório a partir de pesquisa bibliográfica em longe de ser homogêneo, em uma constante correlação de forças em busca de 
referenciais

produzidos legitimidade, na medida em pelas Ciências Humanas, por que tais forças revelam os meio de autores da próprios conflitos de interesses sociologia contemporânea entre seus agentes.

que refletem de forma

crítica sobre como a ciência

atual considera o "social".

Figura 2. Síntese das características dos estudos incluídos na revisão integrativa. João Pessoa (PB), 2019.

Quanto à fonte, observa-se na Figura 2 que os artigos foram publicados nos seguintes periódicos: Revista Ciência \& Saúde, Escola Anna Nery Revista de Enfermagem, Saúde e Sociedade, Revista Acta Paulista de Enfermagem, Revista Brasileira em Promoção da Saúde, Revista Saúde Coletiva, Revista Psicologia - Teoria e Prática, Revista Saúde Pública, Revista Mineira de Enfermagem, Revista de Enfermagem UERJ e Revista de Enfermagem UFSM. Em relação ao idioma, todos foram publicados em português.

No que se refere ao ano de publicação dos artigos, 2011 e 2014 apresentaram maior número de publicações, respectivamente 5 e 4, seguidos dos anos 2012 com 3 publicações, e 2007, 2010, 2013, 2016 e 2017 com uma publicação cada. Quanto ao local de realização dos estudos, todos foram realizados no Brasil. Observa-se, no que diz respeito à metodologia, que $82,4 \%$ das publicações se constituem de estudos com abordagem qualitativa; $11,8 \%$, quantitativa; e 5,8\% quanti-qualitativa. Aponta-se que a maioria dos estudos é do tipo exploratório-descritivo (47\%), seguida de estudos de caso $(11,8 \%)$, um relato de experiência $(5,8 \%)$, uma análise bibliométrica, uma análise crítica da literatura, um estudo transversal, um estudo documental, um levantamento epidemiológico, e um bibliográfico.

Após a leitura exploratória, analisaram-se e interpretaram-se os artigos, sendo então agrupados em uma categoria: determinantes sociais e o acesso dos homens aos serviços de saúde.

\section{DISCUSSÃO}

\section{Determinantes sociais e o acesso dos homens aos serviços de saúde}

Os resultados encontrados constataram que os determinantes sociais são, em grande parte, responsáveis pela iniquidade do acesso aos serviços de saúde. O sexo masculino tem dificuldade em reconhecer suas necessidades voltadas à saúde, e, muitas vezes, prefere mascarar suas fragilidades por entender que o cuidado não é uma prática masculina, não considerando a prevenção da saúde como um critério predominante em suas vidas. Por conseguinte, observou-se a necessidade de os 
homens refletirem sobre a masculinidade para a compreensão dos comprometimentos da saúde do homem.

Independentemente do grau de escolaridade, os homens consideram-se mais fortes e mais ativos do que as mulheres, julgando-as mais frágeis e vulneráveis ao acometimento de doenças, justificando, assim, a presença frequente delas nas unidades básicas de saúde. Afirmam também a ausência de ações específicas voltadas para a saúde do homem; para eles, a única ação da qual todos têm conhecimento é o Novembro Azul, cujo objetivo é conscientizá-los a respeito do câncer de próstata. Contudo, podemos afirmar que a referida ação não alcança 100\% do público-alvo, dificultando, assim, a realização específica de serviços de saúde para o público masculino. A ausência dos homens na utilização das unidades básicas de saúde ocorre por diversos motivos, tais como constrangimento, medo, falta de informação sobre a necessidade de um acompanhamento preventivo de saúde, e até mesmo preconceito sobre a realização do exame. Os artigos A1, A4, A9 e A11 mostram que há uma grande ausência de público masculino nas unidades básicas em busca de saúde preventiva. ${ }^{14,17,22,24}$

Para atingir o objetivo geral de promover melhoria nas condições de saúde da população masculina adulta, a Política Nacional de Atenção Integral à Saúde do Homem - PNAISH - baseou-se em cinco eixos para o seu desenvolvimento, sendo eles: acesso e acolhimento; saúde sexual e reprodutiva; paternidade e cuidado; doenças prevalentes na população masculina; e, por fim, prevenção de violências e acidentes.

Nos artigos A5, A6, A7, A8 e A17, é possível identificar que a criação do PNAISH não foi suficiente para inserir o público masculino no cenário da saúde, havendo lacunas desde a sua criação. A ausência de ações direcionadas ao público masculino faz com que a população não tenha interesse em frequentar os serviços de saúde, pois muitos homens destacam apenas o Novembro Azul como ação preventiva direcionada unicamente a eles. A falta de informação sobre essa política não é de cunho unilateral, pois é possível percebê-la também nos profissionais de saúde. ${ }^{18-}$ 21,30

Evidenciou-se a ausência de capacitação/especialização voltada para a atenção à saúde dos homens. A dificuldade existente entre os profissionais de enfermagem no acolhimento e consulta direcionada ao homem é um problema existente no programa, uma vez que os profissionais de enfermagem geralmente estão direcionados à realização de consultas apenas para a saúde da mulher, sendo desta forma constrangedor tanto para o profissional quanto para o paciente homem falar sobre algo em seus genitais, por exemplo. No artigo A15, é possível perceber a dificuldade existente a partir do acolhimento ao cliente. ${ }^{28}$ A proposta de acolhimento pelo Ministério da Saúde 
significa um novo modo de operar os processos de trabalho em saúde, ouvindo-lhes os pedidos e assumindo, no serviço, o papel de acolher, escutar e pactuar respostas mais adequadas aos usuários.

Nos artigos A12 e A13, observou-se que os determinantes sociais implicam diretamente na participação do homem nos serviços de saúde. Muitos consideram-se os provedores da casa, não podendo faltar ao trabalho para cuidar da saúde, que fica em segundo plano. Percebe-se uma aproximação do homem nos serviços de saúde com o aparecimento do processo de envelhecimento, seja este após um adoecimento ou com o surgimento de doenças crônicas. ${ }^{25,26}$

A ausência de infraestrutura organizacional foi percebida nos artigos A2 e A3, em que os homens relatam não terem um ambiente agradável para recebê-los, havendo constrangimento ao entrar em um ambiente predominantemente feminino para solicitar um atendimento. Cita-se como exemplo os homens com disfunção erétil que por vezes gostariam de saber a origem do problema, mas por vergonha de explaná-lo, realizam a automedicação com Viagra, buscando o serviço de saúde apenas quando o problema se torna grave ou desencadeia outras reações. ${ }^{15,16}$

Por fim, o artigo A16 apresentou deficiências no estudo do âmbito acadêmico direcionado à saúde do homem como um todo, apresentando uma abordagem generalista, superficial, fragmentada e bastante descontextualizada do ambiente sociocultural dos homens, evidenciando esse déficit diretamente pela falta de conhecimento específico sobre a saúde do homem nos serviços de saúde. ${ }^{29}$

Destaca-se que esta pesquisa apresenta limitações, tanto pelo reduzido número de estudos incluídos a partir de fortes evidências científicas, como pela escassa produção de publicações atuais, sendo necessária a ampliação de estudos nessa área.

\section{CONSIDERAÇÕES FINAIS}

O estudo revelou que a compreensão dos determinantes sociais enquanto barreiras socioculturais e institucionais é um fator importante para a proposição de estratégias e medidas a fim de promover o acesso dos homens aos serviços de atenção primária. As produções científicas online evidenciaram o desafio de não somente incluir o homem nas ações realizadas pela UBS, mas, principalmente, de sensibilizá-lo quanto à importância do cuidado.

A atenção à saúde do homem e a seus determinantes sociais necessita de ações intersetoriais que visem à melhoria da qualidade de vida e saúde. Essas ações devem estar pautadas na participação social, na promoção da autonomia do homem e na atuação dos profissionais envolvidos na efetivação dessa política. A melhoria da saúde do homem ainda é um grande desafio, visto que 
requer ações de infraestrutura e funcionamento de serviços de saúde, educação em saúde específica à população masculina, bem como reeducação permanente dos profissionais da área.

\section{CONTRIBUIÇÕES}

Informa-se que todos os autores contribuíram igualmente na concepção do projeto de pesquisa, coleta, análise e discussão dos dados, bem como na redação e revisão crítica do conteúdo com contribuição intelectual e na aprovação da versão final do estudo.

\section{CONFLITO DE INTERESSES}

Nada a declarar.

\section{REFERÊNCIAS}

1. Nunes-Czorny R, Gazetta C, Pinto M, Mendonça-Ribeiro R, Beretta D, Rodrigues C. Perfil do usuário homem atendido em uma unidade básica de saúde da família. Revista de Enfermagem UFPE on line [Internet]. 2017 Mar 7; [cited 2021 Jun 11]; 11(4): 1624-1631. Available from: https://periodicos.ufpe.br/revistas/revistaenfermagem/article/view/15231

2. Rodrigues-Miranda H, Aguiar-Corrêa J, Vidal-Nogueira L, Proença-Palmeira I, Leal-AtaídeRodrigues I. Desvendando saberes e preocupações sobre a saúde entre homens ribeirinhos. Revista de Enfermagem UFPE on line [Internet]. 2017 Ago 22; [cited 2021 Jun 11]; 11(9): 3446-3453. Available from: https: //periodicos.ufpe.br/revistas/revistaenfermagem/article/view/110244

3. Ministério da Saúde. Fortalecimento da Política Nacional de Atenção Integral à Saúde do Homem ( PNAISH ): compromisso versus ação na atenção básica [Internet]. Brasilia: Ministério da Saúde; 2013. 89 p. Available from: https://www.saude.gov.br/images/pdf/2014/maio/21/CNSHDOC-Fortalecimento-da-PNAISH.pdf

4. Bastos GAN, Santos IS, Costa JSD, Capilheira MF. Uso de serviços ambulatoriais nos últimos 15 anos: comparação de dois estudos de base populacional. Rev. bras.epidemiol. [Internet]. 2011 Dec [cited $2020 \mathrm{Apr}$ 17]; 14 (4): 620-632. Available from: https://doi.org/10.1590/S1415790X2011000400009.

5. Organização Mundial de Saúde. A conceptual framework for action on the social determinants of health. Geneva: Orgavização Mundial de Saúde; 2010.

6. Carrapato P, Correia P, Garcia B. Determinante da saúde no Brasil: A procura da equidade na saúde. Saude e Soc [Internet]. 2017; 26(3):676-89. Available from: https://doi.org/10.1590/S0104$\underline{12902017170304}$

7. Gomes R, do Nascimento EF, de Araújo FC. Por que os homens buscam menos os serviços de saúde do que as mulheres? As explicações de homens com baixa escolaridade e homens com ensino superior. Caderno de Saúde Pública. 2007 Mar;23(3):565-574. Available from: https://www.scielosp.org/article/csp/2007.v23n3/565-574/pt/ModalArticles.

8. Andrade LOM, Barreto ICHC, Bezerra RC. Atenção primária à saúde e estratégia saúde da família. In: Campos GWS, Minayo MCS, Akerman M, Drumond Jr. M, Carvalho, YM, organizadores. Tratado de saúde coletiva. São Paulo: Hucitec; 2008. p. 783-836. 
9. Couto MT, Pinheiro TF, Valença O, Machin R, Silva GSN da, Gomes Rl. O homem na atenção primária à saúde: discutindo (in)visibilidade a partir da perspectiva de gênero. Interface (Botucatu) [Internet]. 2010 June [cited 2020 Apr 17]; 14(3): 257-270. Available from: http: / / www.scielo.br/scielo.php?script=sci_arttext\&pid=S1414-

$32832010000200003 \&$ lng $=$ en. https://doi.org/10.1590/S1414-32832010000200003.

10. Figueiredo W. Assistência à saúde dos homens: um desafio para os serviços de atenção primária. Ciênc. saúde coletiva [Internet]. 2005 Mar [cited 2020 Apr 17] ; 10(1): 105-109. Available from: http: //www.scielo.br/scielo.php?script=sci_arttext\&pid=S1413$81232005000100017 \&$ Ing $=$ en. https://doi.org/10.1590/S1413-81232005000100017.

11. Polit DF, Beck CT. Fundamentos de Pesquisa em Enfermagem. 8th ed. Porto Alegre: Artes Médicas; 2015.

12. Beck CLC, Lisbôa RL, Tavares JP, da Silva RM, Prestes FC. Humanização da assistência de enfermagem: percepção de enfermeiros nos serviços de saúde de um município. Revista Gaúcha de Enfermagem. 2009 Mar; 30(1):54 - 61. Available from: https://www.seer.ufrgs.br/RevistaGauchadeEnfermagem/article/view/5102/6561.

13. Bervian PA, Cervo AL. Metodologia Científica. São Paulo: Prentice Hall; 2002

14. Knauth DR, Couto MT, Figueiredo WS. A visão dos profissionais sobre a presença e as demandas dos homens nos serviços de saúde: perspectivas para a análise da implantação da Política Nacional de Atenção Integral à Saúde do Homem. Ciênc. saúde coletiva [Internet]. 2012 Oct [cited 2020 Apr 17] ; 17( 10 ): 2617-2626. Available from: http: / / www.scielo.br/scielo.php?script=sci_arttext\&pid=S1413-

$81232012001000011 \&$ lng $=$ en. https://doi.org/10.1590/S1413-81232012001000011.

15. Silva PAS, Furtado MS, Guilhon AB, Souza NVDO, David HMSL. A saúde do homem na visão dos enfermeiros de uma unidade básica de saúde. Esc. Anna Nery [Internet]. 2012 Sep [cited 2020 Apr 17] ; 16(3): 561-568. Available from: http: / /www.scielo.br/scielo.php?script=sci_arttext\&pid=S1414-

81452012000300019\&lng=en. https://doi.org/10.1590/S1414-81452012000300019.

16. Barbiani R, Junges JR, Nora CRD, Asquidamini F. A produção científica sobre acesso no âmbito do Sistema Único de Saúde do Brasil: Avanços, limites e desafios. Saude e Soc [Internet]. 2014; 23(3):855-68. Available from: https://doi.org/10.1590/S0104-12902014000300010

17. Fontes WD de, Barboza TM, Leite MC, Fonseca RLS, Santos LCF dos, Nery TCL. Atenção à saúde do homem: interlocução entre ensino e serviço. Acta paul. enferm. [Internet]. 2011 [cited 2020 Apr 17]; 24(3):430-433. Available from:

http: / /www.scielo.br/scielo.php?script=sci_arttext\&pid=S0103-

21002011000300020\&lng=en. https://doi.org/10.1590/S0103-21002011000300020.

18. Cavalcanti JRD, Ferreira JA, Henriques AHB, Morais GSN, Trigueiro JVS, Torquato IMB. Assistência Integral a Saúde do Homem: necessidades, obstáculos e estratégias de enfrentamento. Esc. Anna Nery [Internet]. 2014 Dec [cited 2020 Apr 17] ; 18(4):628-634. Available from: http: / / www.scielo.br/scielo.php?script=sci_arttext\&pid=S1414-

81452014000400628\&lng=en. https://doi.org/10.5935/1414-8145.20140089.

19. Carrapato P, Correia P, Garcia B. Determinante da saúde no Brasil: A procura da equidade na saúde. Saude e Soc [Internet]. 2017; 26(3):676-89. Available from: https://doi.org/10.1590/S0104- 
20. Rocha CLM, Paz ASM, Macena HMR, Brasileiro VT. Atenção integral à saúde do homem: um desafio na atenção básica. Rev Bras em Promoção da Saúde [Internet]. 2016; 29(4):554-63. Available from: https://periodicos.unifor.br/RBPS/article/view/5301

21. Marchiori BP, Pelegrini Filho A. A Saúde e seus Determinantes Sociais. Rev Saúde Coletiva [Internet]. 1983; 17(2):32-6. Available from: https: / / www.scielosp.org/article/physis/2007.v17n1/77-93/\#ModalArticles

22. Separavich MA, Canesqui AM. Men's health and masculinities in the Brazilian Comprehensive Healthcare Policy for Men: A bibliographical review. Saude e Soc [Internet]. 2013; 22(2):108-20. Available from: https://doi.org/10.1590/S0104-12902013000200013

23. Silva RP. Gênero e Saúde: o cuidar do homem em debate. Psicol Teor e Prática [Internet]. 2011; 13(3):152-66. Available from:

http://editorarevistas.mackenzie.br/index.php/ptp/article/view/3040

24. Albuquerque GA, Leite MF, Belém JM, Nunes JFC, Oliveira MA de, Adami F. O homem na atenção básica: percepções de enfermeiros sobre as implicações do gênero na saúde. Esc. Anna Nery [Internet]. 2014 Dec [cited 2020 Apr 17] ; 18(4): 607-614. Available from:

http: / /www.scielo.br/scielo.php?script=sci_arttext\&pid=S1414-

81452014000400607\&lng=en. https:// doi.org/10.5935/1414-8145.20140086.

25. Silva MV, Nobre de AA. A Política Nacional de Saúde do Homem: necessidade ou ilusão? Rev Psicol Política [Internet]. 2010;10(20):215-26. Available from: https: / / dialnet.unirioja.es/servlet/articulo?codigo $=4000748$

26. Schwarz E, Gomes R, Couto MT, Moura EC de, Carvalho S de A, Silva SFC da. Política de saúde do homem. Rev. Saúde Pública [Internet]. 2012 Dec [cited 2020 Apr 17]; 46( Suppl 1 ): 108-116. Available from: http://www.scielo.br/scielo.php?script=sci_arttext\&pid=S003489102012000700015\&lng=en. Epub Dec 11, 2012. https://doi.org/10.1590/S003489102012005000061.

27. Santana EN de, Lima EM de M, Bulhões JLF, Monteiro EMLM, Aquino JM de. A atenção à saúde do homem: ações e perspectivas dos enfermeiros. Rev Min Enferm [Internet]. 2011;15(3):324-32. Available from: http://www.reme.org.br/artigo/detalhes/41

28. Ribeiro DB, Terra MG, Jeanninne A, Lacchinni B, Camponogara S, Beuter M. Saúde dos homens: abordagem na formação de enfermeiros. Rev Enferm UERJ [Internet]. 2014;22(4):540-5. Available from:

https: //www.e-

publicacoes.uerj.br/index.php/enfermagemuerj/article/view/15398

29. Ribeiro DB, Terra MG, Jeanninne A, Lacchinni B, Camponogara S, Beuter M. Saúde dos homens: abordagem na formação de enfermeiros. Rev Enferm UERJ [Internet]. 2014;22(4):540-5. Available from:

https: //www.e-

publicacoes.uerj.br/index.php/enfermagemuerj/article/view/15398

30. Garbois, Júlia Arêas, Sodré, Francis e Dalbello-Araujo, MaristelaDeterminantes sociais da saúde: o “social” em questão1 . Saúde e Sociedade [internet]. 2014, v. 23, n. 4 [cited 2021 Jun 12 ] , pp. 1173-1182. Available from: https://doi.org/10.1590/S0104-12902014000400005. 


\section{Correspondência}

Jéssica Lorena Palmeira de Morais

E-mail: jessicapalmeira@hotmail.com

Submissão: $12 / 08 / 2020$

Aceito: 10/06/2021

Copyright $\odot 2021$ Revista de Enfermagem UFPE on line/REUOL.

(cc) Es Este é um artigo de acesso aberto distribuído sob a Atribuição CC BY 4.0 Creative Commons AttributionShareAlike 4.0 International License, a qual permite que outros distribuam, remixem, adaptem e criem a partir do seu trabalho, mesmo para fins comerciais, desde que lhe atribuam o devido crédito pela criação original. É recomendada para maximizar a disseminação e uso dos materiais licenciados. 